\title{
La productividad del recurso humano, factor estratégico de costos de producción y calidad del producto: Industria de confecciones de Bucaramanga
}

\section{Human resource productivity, strategic factor of production costs and quality of product: Clothing industry in Bucaramanga}

\section{Ofelia Gómez Niño}

Contadora Pública, magíster en Administración de Empresas. Docente investiga-

dora de las Unidades Tecnológicas de Santander. Bucaramanga, Colombia.

ofeliagmz@yahoo.com.

Clasificación del artículo: Investigación (Recreaciones)

Fecha de recepción: 31 de marzo de 2011

Fecha de aceptación: 29 de agosto de 2011

Palabras clave: Calidad del producto, competitividad, costos de producción, estrategia, productividad, recurso humano.

Keywords: Product quality, competitiveness, production costs, strategies, productivity, human resources.

\section{RESUMEN}

Este documento resume los resultados de la investigación realizada en las empresas de la industria de confecciones infantiles de Bucaramanga, cuyo propósito se orientó a analizar las variables: valoración del recurso humano en función de los insumos y elementos del costo, productividad del personal vinculado como estrategia de costos y calidad del producto; todo ello en función a estrategias de liderazgo en costos bajos y diferenciación.

La metodología aplicada responde a un estudio de tipo descriptivo con una población de 224 empresas, de las que se tomaron 52 para ser estudiadas. Estas fueron seleccionadas a través de un mues- treo no aleatorio por juicio y conveniencia bajo los siguientes criterios: empresas de la actividad confecciones infantiles registradas y renovadas en la Cámara de Comercio de Bucaramanga en el año 2010 y ubicadas en los diferentes puntos de concentración de la industria, la información fue suministrada directamente por propietarios o gerentes que participaron en el estudio.

La información se obtuvo de fuentes primarias y secundarias. Para la recolección de fuentes primarias se aplicó un instrumento con 32 preguntas relacionadas con las variables por estudiar, información que fue analizada a través de herramientas estadísticas y tecnológicas. 


\section{re-creaciones}

La investigación evidenció que el recurso humano incide significativamente en la generación de valor, productividad y minimización de costos en estas empresas. Por tanto, la fuerza laboral se convierte en un factor estratégico de la gerencia para lograr productos de mejor calidad, diferenciados y a costos atractivos para incursionar y mantenerse en el mercado.

\section{ABSTRACT}

This document summarizes the results of research in companies infant apparel industry in Bucaramanga, whose purpose was aimed at analyzing the variables: assessment of human resources in terms of inputs and cost elements, staff productivity linked as a strategy of cost and product quality, all according to strategies of low cost leadership and differentiation.

The methodology serves a descriptive study with a population of 224 companies, of which 52 were taken for study. These were selected through a non- random sampling by trial and convenience under the following criteria: business activity registered children's clothing and renewed the Chamber of Commerce of Bucaramanga in 2010 and located in different points of concentration of industry. The information was provided directly by owners or managers who participated in the study.

The information was obtained from primary and secondary sources. For the collection of primary sources was used an instrument with 32 questions related to the variables studied, information was analyzed through statistical tools and technologies.

The research showed that significantly affects the human resource in the generation of value, productivity and minimizing costs in these companies. Therefore, the workforce becomes a strategic management to achieve better quality products, differentiated and cost attractive to venture and stay on the market.

\section{INTRODUCCIÓN}

La dinámica del mundo actual exige mayor productividad y competitividad a las organizaciones para incursionar en los mercados y lograr posicionamiento. De esta manera, las organizaciones de hoy deben aplicar mayores esfuerzos en los procesos productivos, para lograr bienes y servicios que agreguen valor para los clientes e inversionistas, a través de estrategias relacionadas con la calidad, atributos de diferenciación, costos bajos y margen de contribución financiera.

Ahora mismo "la productividad es entendida como la relación entre lo que se produce y los recursos humanos, naturales y de capital empleados para producirlo. Una mayor productividad en el uso de los recursos de un país es determinante para aumentar el ingreso nacional per cápita. En el mismo sentido, se entiende por competitividad de un país o de una región la capacidad de producir bienes y servicios que compitan exitosamente en mercados

globalizados, generen crecimiento sostenido en el largo plazo y contribuyan de esa manera a mejorar los ingresos y la calidad de vida de sus habitantes. La competitividad está relacionada con múltiples factores que condicionan el desempeño de las actividades productivas, como la infraestructura, los recursos humanos, la ciencia y la tecnología, las instituciones, el medio ambiente y el entorno macroeconómico" [1].

De todo ello se deriva que la tecnología, la información y el conocimiento cambian de manera acelerada, razón por la cual las empresas deben entrar en la dinámica de la renovación, reconocer las limitaciones y buscar alternativas de solución. Por todo lo anterior, el direccionamiento de las empresas no es tarea fácil, es cuestión de talento, método y estrategia para lograr los objetivos de la organización; sin embargo, no siempre se cuenta con las mismas posibilidades, como es el caso de las micro y pequeñas empresas que trabajan con muy pocos recursos; en consecuencia, quedan en 
posición de desventaja frente a organizaciones más estructuradas y con mejores condiciones.

De acuerdo con el estudio Monitor [2], cuyo propósito fue el de identificar fortalezas y debilidades del departamento de Santander con respecto a la dinámica de la productividad y la competitividad; el capital humano se constituye como factor de competitividad. A su vez, este estudio resalta la necesidad de mano de obra capacitada, a pesar de la creencia en la calidad de los recursos humanos, las instituciones no están preparadas para satisfacer las necesidades del mercado en cuanto a este recurso. En ese momento, tal afirmación responde a la percepción de los empresarios de Bucaramanga, con respecto a la falta de proveedores de tecnología y de mano de obra, aspectos que fueron analizados en dicho estudio.

De igual forma, se analizó la productividad laboral, entendida como el valor agregado por empleado, y concluyó, que uno de los elementos que explica el crecimiento de la economía santandereana es la tasa superior de crecimiento de la productividad laboral comparada con otros departamentos colombianos, la cual viene creciendo a un ritmo del $(6 \%)$ en relación con Antioquia y Valle. Por consiguiente, se deduce que las empresas en el desarrollo de los procesos pueden verse afectadas e incidir en la productividad al carecer del recurso humano altamente calificado.

Otro factor que incide en la productividad del sector de las confecciones se relaciona con la tecnología, la cual es un poco incipiente. En consecuencia los procesos de producción en esta industria son en gran parte artesanales, lo que implica que se requiera suficiente fuerza de trabajo, alto conocimiento y experiencia, organización de las tareas, técnicas y métodos adecuados para la optimización de los recursos; el ideal es contar con los medios y el personal calificado con atributos y capacidades necesarias. No obstante, estas condiciones son difíciles de lograr porque el recurso humano es escaso o poco capacitado, principalmente en las micro y pequeñas empresas de confecciones infantiles, que por no contar con suficiente capacidad instalada deben subcontratar todas las operaciones o partes de ellas, con pequeños talleres satélites.
Adicional a esto, a la hora de ejecutar las actividades productivas, el personal enfrenta limitaciones relacionadas con la formación y experiencia que hacen que las competencias con las que cuentan, no sean las suficientes para facilitar el aprovechamiento óptimo de los recursos de producción. De ahí que la productividad se vea afectada y conlleve a la elevación de los costos de fabricación, y en consecuencia se fijen precios de venta superiores con un margen de contribución menor. Por esta razón, se consideró importante analizar las estrategias que aplica la industria de confecciones infantiles de Bucaramanga, con el objeto de proponer tácticas que permitan mejorar la administración del recurso humano y prever el incremento de los niveles de productividad, con miras a obtener mejor manejo del costo de la mano de obra vinculada a la producción en estas organizaciones.

En el presente estudio se identificaron aspectos importantes que conllevan a plantear una propuesta de intervención que fortalezca la industria en lo relacionado a costos de producción, calidad de los productos y a la aplicación de estrategias que mejoren los procesos de producción en cuanto a la optimización de los recursos utilizados, la productividad y alcance del liderazgo es importante para impulsar la competitividad en el mercado de estas empresas.

Como se mencionó anteriormente, los negocios de este sector de la industria son carentes de estructuras de costos técnicamente elaboradas, por tanto los costos de la mano de obra no son bien administrados, aspectos que permitieron a las Unidades Tecnológicas de Santander iniciar una segunda fase en el proceso de investigación, con el propósito de diseñar una estructura de costos adecuada en lo referente a las condiciones en las que operan las micro y pequeñas empresas de confecciones de Bucaramanga, junto con el diseño de un software que facilite el manejo de los costos y la toma de decisiones entre las que se considera la administración del recurso humano como factor de productividad, calidad y costo.

\section{METODOLOGÍA}

La investigación fue de tipo descriptivo, su principal propósito se centró en observar el objeto de 
estudio para especificar: características, estructura y dinámica de las empresas de la industria de las confecciones infantiles de Bucaramanga, con el fin de recolectar, medir y analizar la información útil para estudiar dicho objeto y cumplir con los objetivos propuestos [3].

\subsection{Población y muestra}

La población estuvo representada por 224 empresas registradas y renovadas en la Cámara de Comercio de Bucaramanga en el año 2010, dato que se obtuvo de la base de datos de empresas de confecciones infantiles inscritas en el registro mercantil; de estas se tomaron 52 para ser observadas, las cuales representan el $23 \%$ de la población total. El muestreo fue no aleatorio por juicio y conveniencia, teniendo en cuenta los siguientes criterios de selección: ubicación geográfica de los principales puntos de concentración productiva, empresas inscritas y renovadas en la Cámara de Comercio de la región, año 2010 y empresas dedicadas a la industria de confección infantil [4]. Asimismo, se tuvo en cuenta en la selección de las empresas estudiadas el tamaño y trayectoria de las mismas, de modo que se incluyeran grandes, medianas, pequeñas y micro empresas, con alguna experiencia en el mercado.

El número de empresas por observar se determinó a partir de la Ec. 1.

$$
n=\frac{n_{o}}{1+\frac{n_{o}}{N}}
$$

$\mathrm{N}$ : Población

$\mathrm{n}$ : Tamaño de la muestra

$\mathrm{n}_{0}$ : Primera aproximación al tamaño de la muestra

Z: Nivel de confianza

P: Proporción de elementos de la población que tienen la característica que se está examinando

Q: Proporción de elementos de la población que no tienen la característica que se está examinando

d: Margen de error

Para desarrollar la fórmula anteriormente expuesta se hace necesario tener el valor de n 0 la que se obtiene, a partir de la implementación de la Ec. 2.

$$
n_{o}=\frac{z^{2} P Q}{d^{2}}
$$

Cuando no se conoce la proporción poblacional se puede tomar el valor de $\mathrm{P}$, igual a 0,5 y por tanto el de $\mathrm{Q}$, igual a 0,5 , lo que hace que el tamaño de la muestra sea lo suficientemente grande para garantizar como mínimo la precisión requerida en el estudio.

Para el caso de las fábricas de confecciones infantiles de Bucaramanga, la información con la que se determinó la muestra fue la siguiente:

$\mathrm{N}$ : 224 empresas (fábricas registradas y renovadas periodo 2010, Cámara de Comercio de Bucaramanga) [5]

Z: Nivel de confianza, se fijó en el 90\% (según tabla, 1,645)

d: Margen de error del muestreo, se estableció en un $10 \%(0,1)$

P: Proporción de elementos de la poblacional que tiene las características $(0,5)$

Q: Proporción de elementos de la poblacional que no tienen las características $(0,5)$

$$
\begin{aligned}
& n_{o}=\frac{(1,645)^{2}(0,5)(0,5)}{(0,1)^{2}} \\
& n_{o}=67,650625
\end{aligned}
$$

La primera aproximación a la muestra es de 67,650625 y a partir de este valor se determinó la muestra que corresponde a:

$$
\begin{aligned}
& n=\frac{67,650625}{1+\frac{67,650625}{224}} \\
& n=51,9585
\end{aligned}
$$

\subsection{Fuentes de información}

El estudio se soportó en fuentes primarias y secundarias.

\subsubsection{Fuentes primarias}

Se diseñó un instrumento en la modalidad de en- 
cuesta con 32 preguntas orientadas a obtener información relacionada con las principales variables para estudiar: valoración del recurso humano en función de los insumos y elementos del costo, productividad del personal vinculado como estrategia de costos y calidad del producto; todo ello en función de las estrategias de liderazgo en costos bajos y diferenciación. El instrumento se aplicó mediante la visita a las empresas seleccionadas en la muestra, la información obtenida fue suministrada directamente por los dueños o gerentes.

\subsubsection{Fuentes secundarias}

Se hizo una revisión documental sobre estudios efectuados en las empresas del sector, se consultaron y se verificaron datos en la Cámara de Comercio de Bucaramanga, se examinó información de gremios y se consultaron referencias bibliográficas: artículos especializados, libros, revistas, entre otros.

\subsection{Análisis de la información}

Los datos fueron procesados y analizados mediante la aplicación y uso de herramientas estadísticas y hojas de Excel.

\subsection{Fuente de financiación}

Los recursos relacionados con espacio físico, herramientas tecnológicas, base de datos, medio de transporte para trabajo de campo y costo del recurso humano vinculado al proyecto de investigación, fueron suministrados en su totalidad por las Unidades Tecnológicas de Santander, por tanto los recursos de financiación fueron generados por el Estado a través de esta institución.

\section{RESULTADOS}

En el entorno de las organizaciones, la variable costos tiene una gran significancia por la complejidad con la que debe ser administrada para alcanzar un buen desempeño financiero como resultados de la productividad de los recursos involucrados en los procesos, de manera que se logre la competitividad en los mercados.

Ahora bien, la gestión de los administradores en las organizaciones es medida por el valor agregado que generen en función del cliente y de los inversionistas. En esta dinámica la productividad juega un papel muy importante porque ella permite optimizar los recursos y minimizar los costos. En este sentido es fundamental el aporte del recurso humano vinculado a los procesos de producción, puesto que su capacidad productiva conlleva a mejorar el costo de los productos fabricados en la industria de las confecciones infantiles de la región.

Desde este punto de vista las empresas deben "acabar las prácticas de hacer negocios sobre la base del precio, en vez de ello deben minimizar el costo total; mejorar constantemente y siempre el sistema de producción y servicio para mejorar la calidad y la productividad, y así reducir los costes continuamente. Otro de los aspectos propuestos es el de implantar la formación para el trabajo y el liderazgo. El objetivo de la supervisión debería consistir en ayudar a las personas, a las máquinas y aparatos para que hagan un trabajo mejor" [6], por consiguiente las buenas prácticas conducen a mejores resultados dentro de la industria.

Bajo esta premisa, las empresas de confecciones centran la atención en el recurso humano y en factores que permitan fortalecer los procesos de producción para lograr un mejoramiento de los costos. Disminuir los costos sin afectar la calidad de los productos es el ideal; sin embargo, esto implica organizar el trabajo, buscar métodos de optimización para hacer que las operaciones se logren con el máximo de eficiencia y el mínimo error en calidad. La tecnología es otro de los factores que contribuye con los costos de producción, entendida esta no solo como el uso de herramientas mecanizadas o computarizadas, sino como el conocimiento aplicado a los procesos en el que las personas son gestoras a partir de la creatividad e ingenio.

Es preciso resaltar que en el mundo actual las personas tiene gran reconocimiento por el activo intangible que poseen, ese gran potencial permite agregar valor para los clientes e inversionistas en la dinámica empresarial; además se convierte en factor competitivo para las organizaciones por ser fuente de riqueza patrimonial. Todo ello impulsa 


\section{re-creaciones}

la consolidación, desarrollo y crecimiento de las empresas y el fortalecimiento de la economía.

En tal sentido el capital humano permite a las empresas rediseñar los procesos, con el conocimiento y experiencia; capacidades que le sirven al individuo para innovar y mejorar la forma de realizar el trabajo, por tanto el conocimiento puede provenir de diversas fuentes, desde mejoras en la calidad y productividad, ideas del personal de ventas para ofrecer un mejor servicio al cliente, nuevos diseños técnicos o científicos, la propia cultura de la organización, el talento directivo. Aunque estos elementos son más difíciles de identificar y percibir, puesto que no tienen entidad material y no son susceptibles de tocarse o percibirse de un modo preciso. Tales recursos se engloban bajo la denominación de intangibles [7].

Es en este contexto que se precisa " una definición más amplia de recursos intangibles que ha sido desarrollada a través de diversos modelos para medir el capital intelectual, lo cual nos lleva, necesariamente a estructurar una primera gran división entre el capital humano y el capital estructural", como se observa en el Fig. 1 [8]. En que el problema radica en cómo medir ese conocimiento, considerado este como la nueva base de la riqueza y a su vez, en cómo redefinir las cuestiones de la empresa en lo que se refiere al capital humano. Es así, como este aporta la productividad como factor de costo, elemento que contribuye con el desempeño financiero y el crecimiento de la organización.

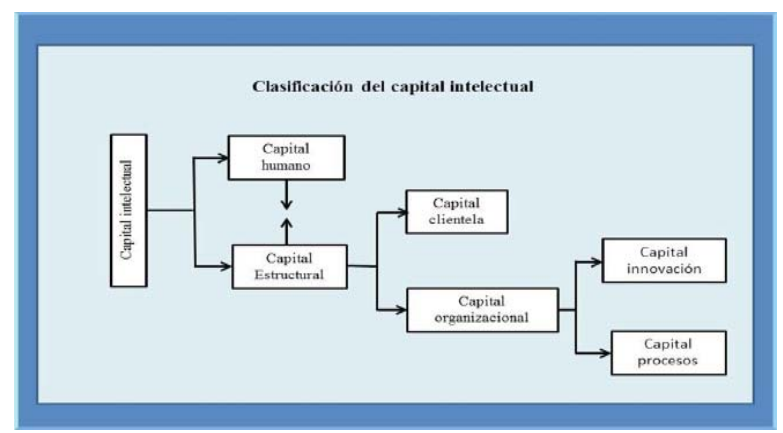

Fig.1. El capital intelectual.

Fuente: Revista Contabilidad \& Contaduría, p. 152.

\subsection{El recurso humano como factor de costo}

La elaboración de un producto en la industria de confecciones requiere llevar a cabo un proceso de producción en el que se integran una serie de recursos e insumos, los cuales son transformados bajo una estructura sistémica y organizada por operaciones secuenciales o simultáneas, entre las que se destacan: el diseño, trazo, corte, ensamble, terminado y empaque; en cada una de ellas se hace necesaria la fuerza de trabajo del recurso humano. Contar con personal calificado garantiza el desarrollo de los procesos en condiciones óptimas, de tal forma que la productividad de este recurso permita minimizar costos y mejorar calidad.

Cabe señalar que las 52 empresas que sirvieron como unidad de observación en el estudio realizado, evidencian la relevancia de mantener personal calificado en cada una de las operaciones, por ser el recurso humano elemento fundamental en el costo de producción, como se muestra en la Fig. 2. Por tal razón en la medida en que las empresas tengan una buena selección de personal, diseñen programas de capacitación para operaciones propias de la organización acordes con los cambios generados por los sistemas de información, la tecnología y la sociedad del conocimiento y logren mantener este recurso, es posible incrementar la productividad y la diferenciación de los productos fabricados. De otra, parte es pertinente propiciar un ambiente organizacional adecuado en el que el personal actúe de manera libre y adquiera alto grado de compromiso y responsabilidad, de modo que sean partícipes en el logro de las metas propuestas por la administración.

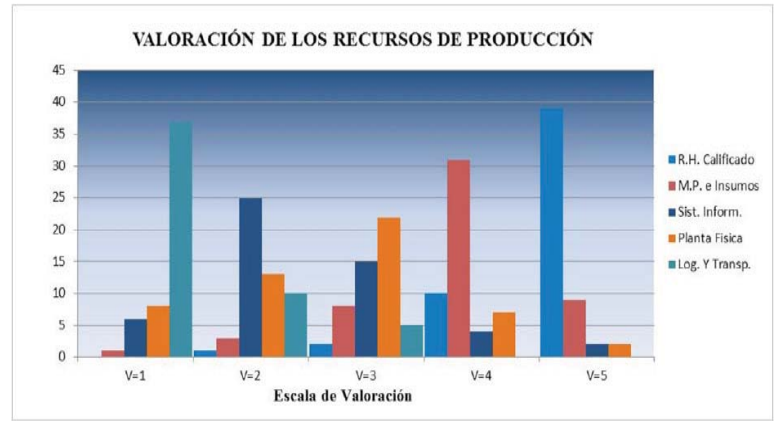

Fig. 2. Valoración de recursos vinculados a la producción. Fuente: información suministrada por empresas observadas 2010 (V: valoración, escala 1-5) 
Lo anterior reafirma la percepción que tienen las 52 empresas de confecciones infantiles que fueron observadas. Al momento de solicitarles que calificaran la importancia que representa los insumos vinculados a los procesos productivos, de acuerdo con el grado de contribución en la elaboración de los productos, 39 de ellas aplican la mayor valoración de la escala, cinco (5) al recurso humano, siendo este el recurso la fuerza que dinamiza el proceso. Al mismo tiempo contemplan la posibilidad de buscar personal calificado o capacitarlo para extraer el mayor potencial en función del mejoramiento de los costos por mano de obra.

\subsection{El costo de producción y los elemen- tos que lo integran}

El costo de producción en la industria es el resultado de una serie de esfuerzos materiales y sacrificios económicos en los que incurre la empresa para desarrollar los procesos de transformación, todos ellos integrados al costo de los productos, siempre y cuando se aplique un sistema de costeo total. En el caso que la empresa tome como política costear solo sobre la base del costo variable se deben excluir los costos fijos, llevándolos como costos del periodo y no como costo del producto; en tal situación el costo de producción es menor debido a que cierto valor monetario se trata como gasto. Esta filosofía de costos depende del enfoque de las estrategias con las que se direccione la organización para buscar factores de competitividad.

Entre los recursos que influyen en el costo de producción está la mano de obra, materias primas e insumos, la tecnología, la planta física y maquinaria, la logística y el transporte. Aunque todos estos recursos son necesarios en los procesos de producción, no todos guardan la misma importancia, como se observa en la Tabla 1. De acuerdo con la percepción que manifiestan los empresarios de la industria de confecciones infantiles es que el recurso humano es el insumo de producción de mayor importancia, de manera que 39 de ellas lo valoran con una calificación de cinco (5), seguido de las materias primas que fueron valoradas por
31 de las empresas con calificación de cuatro (4); lo que indica que estos dos recursos son los que requieren especial atención en este sector de la industria.

Tabla 1. Valoración de los recursos vinculados a la produc ción.

\begin{tabular}{|c|c|c|c|c|c|}
\hline \multirow{2}{*}{$\begin{array}{c}\text { Recursos de } \\
\text { producción }\end{array}$} & \multicolumn{5}{|c|}{ Escala de Valoración } \\
\cline { 2 - 6 } & $\mathbf{V = 1}$ & $\mathbf{V = 2}$ & $\mathbf{V = 3}$ & $\mathbf{V = 4}$ & $\mathbf{V = 5}$ \\
\hline $\begin{array}{c}\text { Recurso humano } \\
\text { calificado }\end{array}$ & 0 & 1 & 2 & 10 & $\mathbf{3 9}$ \\
\hline $\begin{array}{c}\text { Materias primas } \\
\text { e insumos }\end{array}$ & 1 & 3 & 8 & 31 & 9 \\
\hline $\begin{array}{c}\text { Sistemas de } \\
\text { información }\end{array}$ & 6 & $\mathbf{2 5}$ & 15 & 4 & 2 \\
\hline Planta física & 8 & 13 & $\mathbf{2 2}$ & 7 & 2 \\
\hline $\begin{array}{c}\text { Logística y } \\
\text { transporte }\end{array}$ & $\mathbf{3 7}$ & 10 & 5 & 0 & 0 \\
\hline Total empresas & $\mathbf{5 2}$ & $\mathbf{5 2}$ & $\mathbf{5 2}$ & $\mathbf{5 2}$ & $\mathbf{5 2}$ \\
\hline
\end{tabular}

Fuente: información suministrada por las empresas observadas 2010

Aunque las empresas del sector confecciones tienen claro el grado de importancia del personal en cada uno de los procesos y operaciones, reconocen al mismo tiempo que es un recurso escaso y poco capacitado, por lo que deben contratar operaciones con talleres satélites. Desde esta perspectiva, estas organizaciones se deben preocupar por establecer estrategias que se direccionen hacia la búsqueda del mejoramiento de sus puntos débiles y al fortalecimiento de sus áreas fuertes. Asi mismo deben evaluar la disponibilidad de recursos con los que cuentan, e implementar acciones que contribuyan a contrarrestar las limitaciones en las que desarrollan los procesos las diversas áreas de la organización.

Aun así, no siempre la administración está en capacidad de diseñar planes adecuados para redireccionar los negocios y obtener desempeños aceptables. A causa de ello es pertinente referenciar que las compañías occidentales consideran que sus empleados son su activo competitivo más significativo y le proporcionan una buena orientación ge- 


\section{re-creaciones}

neral [6]. De ello se deduce que la administración de personal juega un rol significativo en el manejo del recurso humano, "la moderna ingeniería industrial, que busca adaptar los métodos y procesos de trabajo, las máquinas y equipos, las condiciones ambientales de trabajo (como iluminación, nivel de ruido, temperatura, etcétera) a las condiciones humanas, para que las personas se sientan mejor y sean más productivas" [9]. En consecuencia la administración de personal no debe desconocer la importancia que este recurso tiene en los procesos de producción; en la medida que se logre elevar los niveles de productividad es posible alcanzar la disminución de los costos totales.

Por ello,

La primera estrategia, cada día más común en los años setenta por la difusión del concepto de la curva de experiencia, consiste en alcanzar el liderazgo en costos globales mediante un conjunto de políticas funcionales encaminadas a este objetivo básico. El liderazgo en costo exige la construcción de instalaciones de escala eficiente, la búsqueda vigorosa de reducción de costos a partir de la experiencia, un control riguroso de gastos variables y fijos, evitar las cuentas de clientes menores y minimizar los costos en áreas como investigación y desarrollo, fuerza de ventas, publicidad y otras. Tales objetivos no se cumplirán si los directivos no prestan una atención esmerada al control de costos. En toda la estrategia el tema central lo constituye los costos bajos frente a los de la competencia, pero no deben descuidarse la calidad, el servicio ni otros aspectos [10].

\subsection{Dinámica de la calidad, el costo y la es- trategia}

\subsubsection{La calidad de los productos fabrica- dos}

Si bien es cierto que la calidad no debe ser considerada como un atributo del producto, sí es un factor de competitividad, porque eleva la imagen de la marca. La calidad es entendida como el ideal, lo óptimo para suplir las necesidades y generar una sensación de satisfacción a los consumidores, elementos que motivan al cliente a obtener ese producto y no otro de similares características.

En el mercado de moda infantil la calidad de las prendas es un factor decisivo para definir la compra, esta tiene que ver con textura, diseño, moda, tallas, terminados, colores, servicio, entre otros. Dentro de este contexto, la industria de confecciones infantiles sustenta la calidad de los productos principalmente por la tecnología aplicada en los procesos, entendida la tecnología no como aquellas operaciones mecanizadas o computarizadas, sino como la capacidad del recurso humano de hacer las cosas a través de métodos y mecanismos en los que se apliquen procedimientos previamente estructurados, en donde el conocimiento de las personas elevan la productividad de los procesos y la calidad de los productos. La Fig. 3 muestra la variable tecnología como la más relevante, en función de esta variable..

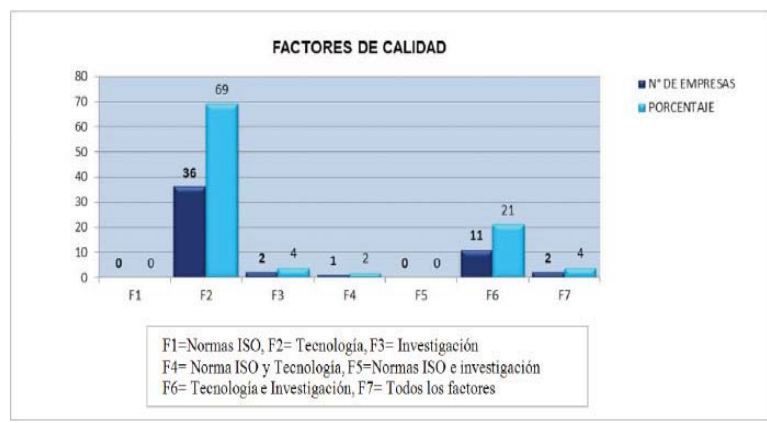

Fig. 3. Factores de calidad.

Fuente: información suministrada por las empresas observadas 2010

En este orden de ideas, se resalta la estrecha relación que guarda el recurso humano con la calidad del producto. Por otra parte, la estrategia también depende de las personas, siendo ellas las que contribuyen para que la empresa logre posicionamiento en el mercado, enfrenten la rivalidad, la competencia y generen mayores beneficios.

La ubicación de una empresa dentro de su sector obedece fundamentalmente a tres factores estratégicos: el liderazgo en costos, la diferenciación $y$ el enfoque [10]. El liderazgo en costos consiste en que una empresa sea el productor de menor costo dentro de su sector industrial; la diferenciación existe cuando una empresa es única por poseer ciertos atributos que para los compradores son muy importantes; el enfoque consiste en la elección de un panorama de competencia estrecho dentro de su sector industrial, este puede ser de 
diferenciación o de liderazgo en costos.

\subsubsection{Los costos del recurso humano vin- culado a la producción}

La administración de los costos en una compañía es un factor determinante del éxito o fracaso, la gerencia no debe descuidar las variables que influyen sobre los mismos. Ahora bien, la producción puede ser altamente manual o automatizada, lo que indica que las personas influyen significativamente cuando es manual, cuando es automatizada la mayor intervención recae sobre las máquinas. En el caso del volumen de producción, este depende en gran medida de la eficiencia con la que se administren estos componentes; cuando se alcanzan altos niveles de eficiencia, bien sea en la mano de obra o en el uso adecuado de la maquinaria, se logra mejorar la productividad y como consecuencia la minimización de costos.

Las empresas de la industria de confecciones infantiles que fueron estudiadas, atribuyen el mejoramiento de los costos a las variables volumen de producción y experiencia del recurso humano, es decir, el 67\% de estas empresas asumen que los costos son menores cuando se incrementa la producción y cuando el personal logra destrezas y capacidades que se evidencian en los niveles de experiencia; como se observa en la Fig. 4.

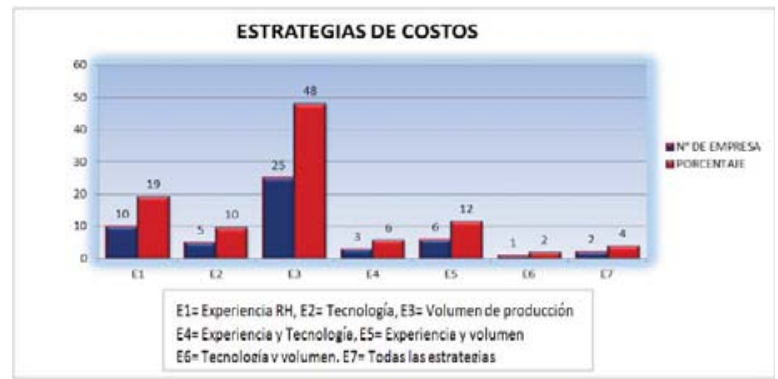

Fig. 4. Estrategias de mejoramiento en costos.

Fuente: información suministrada por las empresas observadas.

El 90\% de las empresas de la muestra fueron micro y pequeñas, con grandes limitaciones para acceder a recursos, con poco costo financiero para tecnificar los negocios; en consecuencia, no les queda otra opción que aprovechar al máximo el personal con el que cuentan y hacer de la producción ma- nual un elemento diferenciador para incursionar en los mercados con productos altamente competitivos.

Aunque estas empresas desarrollan la actividad económica en condiciones muy limitadas, es preciso destacar que han logrado posicionarse en el mercado, porque los productos reúnen las condiciones de diseño, tendencia, calidad y ante todo, manejan costos ajustados a los requerimientos de los clientes. No cabe duda que estas empresas pueden llegar a ser más productivas y competitivas si aplican mejores prácticas administrativas, rediseñan los procesos, hacen un mejor uso de los recursos disponibles y se integran a programas de capacitación e intervención por parte de organismos gremiales, gubernamentales o entes de apoyo empresarial.

En definitiva es importante mencionar que el estudio realizado en las empresas de este sector de la industria santandereana, permitió identificar las fortalezas y debilidades en cada una de las áreas de la organización, aspectos que generan una segunda fase de investigación con el propósito de establecer acciones de intervención para el mejoramiento de la estructura de costos y apoyar el manejo de los mismos a través de una herramienta tecnológica.

\subsubsection{La estrategia como factor de pro- ductividad y competitividad}

Definitivamente, el recurso humano es un factor clave de la productividad y competitividad en los diversos ámbitos organizacionales, como sucede en la industria de confección infantil de Bucaramanga. De esto se deriva que las empresas fijen las estrategias en función de las personas que integran la fuerza laboral y observe detenidamente los aspectos que hacen que estas sean establecidas en forma adecuada, con el fin de que se logren los propósitos para lo cual han sido creadas. De ahí que en la formulación de las estrategias se hace necesario tener en cuenta las capacidades de los empleados. Se observa que para mejorar los procesos y la actuación de cara a los clientes, las ideas deben provenir, cada vez más, de los empleados que están más cerca de los procesos internos y de los clientes de la organización [11]. 


\section{re-creaciones}

Algunos indicadores clave:

- La satisfacción del empleado. Es de suma importancia para la mayoría de las empresas; condición previa para el aumento de la productividad, de la rapidez de reacción, la calidad y el servicio al cliente.

- La retención de los empleados. Objetivo que permite retener aquellos empleados en los que la organización tiene un interés a largo plazo. Cualquier salida no deseada representa una pérdida de capital intelectual del negocio.

- La productividad de los empleados. Es un indicador del resultado del impacto global de haber incrementado las capacitaciones y moral de los empleados, así como la innovación y mejora de los procesos internos y de la satisfacción de los clientes.

Lo anterior hace posible obtener una reducción de costos cuando en los procesos de producción se logra incrementar la productividad manteniendo la calidad, que en buena parte está en manos de quienes realizan el trabajo o hacen uso de los recursos. La productividad conlleva a elevar los niveles de producción con los mismos insumos, es decir, optimizar los recursos para lograr la minimización de costos. En gran medida la productividad depende de la forma como se organice el trabajo, de los métodos, técnicas y procedimientos que aplican las personas en la ejecución de las tareas. Vale decir, que la integración entre el recurso humano, la calidad de los productos y los costos de producción, son una opción estratégica de mejoramiento de la competitividad de las organizaciones, como se observa en la Fig. 5.

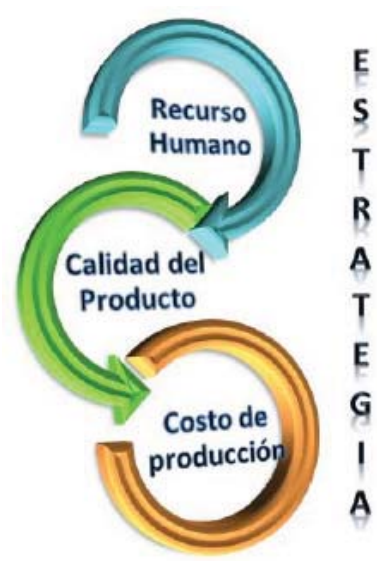

Fig. 5. Estrategias de competitividad.

Las empresas de la industria de confecciones infantiles de Bucaramanga, que participaron en el estudio reconocen la experiencia del personal vinculado a los procesos de producción, siendo esta una ventaja competitiva al elevar los niveles de productividad y competitividad, razón por la cual califican con cinco (5), en la escala de valoración, seguido por la calidad humana y trabajo en equipo, como se muestra en el Fig. 6.

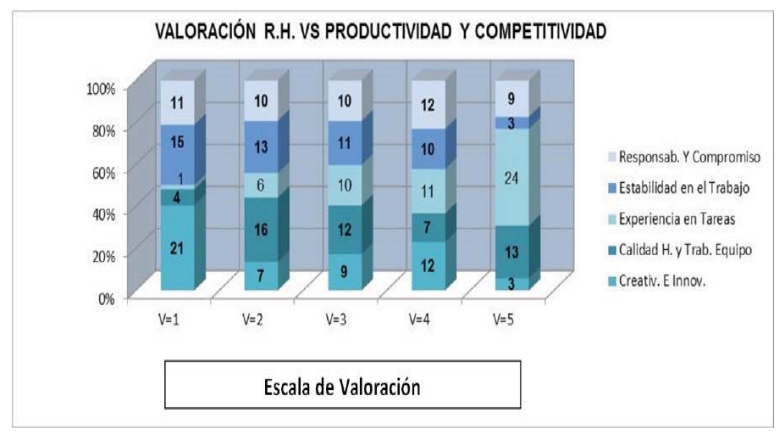

Fig. 6. Valoración del recurso humano.

Fuente: Información suministrada por empresas observadas (V: valoración, escala 1-5).

Al mismo tiempo, es preciso dejar claro que el buen clima laboral y ambiente organizacional generan las condiciones para que las personas exploten sus potencialidades, se motiven a desarrollar el trabajo con calidad, de manera que las empresas se nutran de la creatividad y capacidad de innovación con la que puede mejorar los procesos.

\subsubsection{Innovación en valor estrategia de di- ferenciación y bajo costo}


El valor de un bien es aquel que genera las condiciones para que los consumidores lo adquieran, es a veces entendido en forma errónea como una propiedad o atributo de los bienes o servicios. "El valor es mucho más que una propiedad del objeto: es una ley que gobierna nuestro comportamiento. El valor se incrementa si el desempeño mejora o si el costo se reduce. Algunos métodos incrementan el valor mejorando los bienes o servicios que ofrece la empresa" [12].

Al llegar a este punto es conveniente destacar que la generación de valor para los clientes e inversionistas depende en gran parte de las buenas prácticas de la administración, de manera que las actividades que agregan valor, sean plenamente identificadas.

La innovación en valor se crea en la región en la cual los actos de una compañía inciden favorablemente sobre su estructura de costos y sobre la propuesta de valor para los compradores. Las economías se logran al eliminar y reducir las variables sobre las cuales compite una industria. El valor para los compradores se aumenta al buscar y crear elementos que la industria nunca ha ofrecido. Con el tiempo los costos se reducen todavía más, cuando entran a operar las economías de escala debido al mayor nivel de ventas emanado del valor superior [13].

De lo anteriormente expuesto, es preciso destacar que las empresas de la industria de confecciones infantiles que intervinieron en el estudio, poseen fortalezas que les da ventaja competitiva, pero asi mismo enfrentan debilidades y limitaciones que inciden sobre la productividad y competitividad. En esta dinámica se hace necesario mejorar los procesos productivos, mantener el capital humano con el fin de alcanzar el desarrollo de productos diferenciados, con calidad y a costos totales bajos, que les permita mantenerse en el mercado.

\section{CONCLUSIONES}

Los costos de producción y la calidad de los productos, son una opción estratégica de las empresas de confecciones infantiles de Bucaramanga, para lograr posicionamientos en el mercado. De ahí que estos requieran especial cuidado por parte de la administración, al ser un factor que incide sobre clientes y consumidores que buscan satisfacer las necesidades con productos que posean ciertos atributos y características. Desde esta perspectiva, el recurso humano contribuye en gran medida con la generación de valor para los clientes e inversionistas, elementos que dan ventaja competitiva a estas organizaciones.

Igualmente, el estudio permitió identificar la percepción que tienen estas empresas en relación con el capital humano como factor de productividad, consideran que este es relevante. Resaltan la importancia de contar con recurso humano altamente calificado que posea experiencia y capacidad de trabajo en equipo, competentes para el desarrollo de las actividades; todos estos elementos son determinantes para fortalecer los procesos de producción, aspectos que contribuyen a elevar los niveles de eficiencia. Ahora bien, la creatividad e ingenio del capital humano aporta a la elaboración de productos diferenciados, con calidad y costos favorables.

Por último, se concluye que el capital humano está representado por la fuerza de trabajo, ingenio y creatividad del personal vinculado a la organización. De aquí que se reconozca que las personas son el activo productivo de mayor importancia, este recurso está en función de la productividad, lo cual es posible si se genera un clima laboral y ambiente organizacional apropiados. Aun así, estas empresas se desempeñan en condiciones estrechas, al no contar con estructuras altamente tecnificadas o automatizadas para los procesos, por tanto, les conviene formular opciones estratégicas que propicien las condiciones para capitalizar el recurso humano que tienen disponible.

\section{REFERENCIAS}




\section{re-creaciones}

Productividad y la Competitividad, Bogotá, 2007.

[2] Cámara de Comercio de Bucaramanga, Santander: entorno de negocios competitivos frente al mundo. Centro de Estrategia de Competitividad, UniAndes, 2006.

[3] C. Méndez, Metodología. Diseño y desarrollo del proceso de investigación. México: Mc Graw Hill, 2011.

[4] Cámara de Comercio de Bucaramanga, Empresas inscritas y renovadas en Registro Mercantil. Bucaramanga, 2010.

[5] C. Martínez, Aplicaciones de métodos y técnicas de muestreo. Estadística y muestreo. Colombia: ECOE, pp. 865-868, Bogotá, 2005.

[6] E. Deming, Calidad, Productividad y competitividad. La Salida de la Crisis. España: Díaz Santos, S.A, 1989.

[7] J. Laínez y Y. Fuentes, "Nuevas tecnologías de la información y comunicación: cambios en el sector empresarial". Revis- ta Internacional de Contabilidad \& Contaduría, No 20, Colombia: LEGIS.

[8] M. Rodríguez, "Recursos intangibles e insuficiencia de perspectiva financiera tradicional". Revista Internacional de Contabilidad \& Contaduría, No 21, Colombia: LEGIS, 2004.

[9] I. Chiavenato, Iniciación a la administración de personal. Colombia: Norma, 1997.

[10] M. Porter, Estrategia competitiva. Técnicas para el análisis de los sectores industriales y de la competencia, México: Grupo Patria, 2007.

[11] J. Amaya, Gerencia, planeación y estrategia. Fundamentos, modelos y software de planeación. Prospectiva. Bucaramanga: Universidad Santo Tomás, 2005.

[12] D. Ramírez, Contabilidad Administrativa, México: Mc Graw Hill, 2008.

[13] K. Chan y R. Mauborge, La estrategia del océano azul. Colombia: Norma, 2005. 